\title{
AVERAGE GEOMETRICAL FEATURES OF THE ELECTRON WAVE PACKAGES DISTRIBUTION IN METALLIC CONDUCTORS WITH PULSED AXIAL CURRENT OF HIGH DENSITY
}

Purpose. Calculation and experimental determination of average geometrical features of distributing of macroscopic electron wave packages (EWP) in round cylindrical metallic conductors with the pulsed axial current of high density. Methodology. Theoretical bases of the electrical engineering, bases of atomic and quantum physics, electrophysics bases of technique of high voltage and high pulsed currents. Results. The results of the conducted calculation and experimental researches are resulted on close determination of average geometrical features of distribution of longitudinal and radial EWP of macroscopic sizes in the indicated conductors. These descriptions are included by the average widths of "hot» and "cold» longitudinal and radial areas of conductor, and also average steps of division into the periods of similar areas. Results of the executed calculations and high temperature experiments for average geometrical features of longitudinal EWP in the zincked steel wire of diameter of $1.6 \mathrm{~mm}$ and length of $320 \mathrm{~mm}$ with the aperiodic impulse of current of temporal form $9 \mathrm{~ms} / 160 \mathrm{~ms}$ and by amplitude 745 A coincide within the limits of $19 \%$. Originality. First with the use of methods of atomic and quantum physics the features of the stochastic distributing and mean values of basic geometrical sizes are analysed macroscopic longitudinal and radial EWP in round cylindrical metallic conductors with the pulsed axial current of high density. Practical value. Drawing on the got results in practice will allow more reliably to forecast geometrical sizes and places of localization of arising up in the probed metallic conductors with pulsed axial current of high density longitudinal and radial EWP. References 14, figures 2.

Key words: metallic conductor, pulsed current of high density, electron wave package of conductor, «hot» and «cold» longitudinal and radial areas of conductor, quantization and averaging of geometrical features of electron wave packages of conductor.

Приведены результаты приближсенного выбора усредненного значения $\bar{n}$ целого квантового числа п для мод квантованных волновых пси-функций п-го порядка и квантовомеханического расчета на основе найденного квантового числа $\bar{n}$ усредненных геометрических характеристик квантованных периодических продольных и радиальных волновых электронных пакетов (ВЭП) в круглых сплошных металлических проводниках с большим импульсным аксиальным током. Данные характеристики включают усредненные иирины "горячих» и «холодных» продольных и радиальных участков указанных проводников, формируемых соответствующцми ВЭП, и усредненные шаги периодизации в указанных структурах ВЭП. Выполненные эксперименты на мощцом генераторе длительной апериодической С- компоненты импульсного тока искусственной молнии с амплитудой его плотности до 0,37 кА/мм в оцинкованном стальном проводе радиусом 0,8 мм и длиной 320 мм подтвердили результать выбора для него квантового числа $\bar{n}$ и расчета усредненных ширин «горячих" и «холодных» участков продольных ВЭП, визуально наблюдаемых вдоль интенсивно нагреваемого этим током исследуемого провода за счет слабого рассеяния электронных полуволн де Бройля на атомах кристаллической решетки его металла. Библ. 14, рис. 2.

Ключевые слова: металлический проводник, импульсный ток большой плотности, волновой электронный пакет проводника, «горячие» ихолодные» продольные и радиальные участки проводника, квантование и усреднение геометрических характеристик волновых электронных пакетов проводника.

Introduction. In $[1,2]$ the results of theoretical and experimental studies of wave longitudinal and radial distribution of free electrons in a solid round metal conductor with a high density of pulsed axial current are presented. The results indicate the occurrence of stochastic character in the conductive structure of the conductor radius $r_{0}$ and length $l_{0}>>r_{0}$ containing the electronic quantum number $n$ of half-waves of de Broglie, periodic quantized electron wave packets (EWP) [3]. A distinctive feature of the display data of macroscopic EWP is that they form a relatively «hot» and «cold» longitudinal and radial portions of the conductor, the geometric dimensions of which are defined set forth in [1, 2] laws in wave distributions drifting electrons and the quantized values of the longitudinal $k_{n z}=\pi n / l_{0}$ and radial $k_{n r}=\pi n / r_{0}$ wave numbers $\left(n=1,2,3, \ldots, n_{m}-\right.$ the integer quantum number; $n_{m}$ - the maximum value of the quantum numbers $n$ defined by the principal quantum number $n_{k}$ of the metal atoms of the conductor [3] ). Moreover, the step of the periodic structure of EWP conductor is equal to the sum of the widths of its neighbors «hot» longitudinal $\Delta z_{n h}$ (radial $\Delta r_{n h}$ ) and «cold» longitudinal $\Delta z_{n c}$ (radial $\Delta r_{n c}$ ) sites. However, according to $[2,4]$ temperature levels Joule heating of «hot» and «cold» longitudinal and radial sections of EWP of the conductor may differ by 3.5 times. Data in [2] show that the quantum number $n_{m}=2 n_{k}^{2}$ for copper, zinc and steel conductors $\left(n_{k}=4\right.$ [3]) is 32, which indicates the possibility of having their internal conductive structure 32 modes of longitudinal $\Psi_{n z}(z, t)$ and radial $\Psi_{n r}(r, t)$ of the wave functions. This superposition (overlap) of the quantized wave psi-function of the 
$n$-th order and forms the conductor of its macroscopic quantized longitudinal and radial EWP. Each quantum number $n$ will match their EWP placed along $l_{0}$ length or $r_{0}$ radius of the conductor. Mode indicator (number $n$ ) for said quantized wave psi-function determined by the energy state of the electrons in a conductor-free time of the application to it of electrical voltage and started flowing through it conduction current [5]. The probabilistic nature of the occurrence in metallic conductors with electric current phenomenon quantized periodic macrolocalization free electrons when they drift [5] and, accordingly, the emergence in these conductive materials quantized longitudinal and radial EWP with $n \leq 32$ greatly complicates the prediction of a picture of a periodic distribution in it of indicated EWP. Certain decisions of the electrophysical an issue of the quantum wave nature and importance electrotechnological value at the reference (pilot) evaluation of the results of local action high discharge pulse current to the current-carrying part of the wires (cables) power circuits of powerful highvoltage test of electrical installations [6], as well as shock pulse short circuit [4, 7], and a lightning [8] on the current-carrying parts of the power circuit of electric power facilities, will contribute to the knowledge of the average quantum number $n$ values (the value $\bar{n}$ ) and, accordingly, average geometric characteristics of the quantized periodic longitudinal and radial EWP in round metallic conductors with pulsed axial current $i_{0}(t)$ of high density.

The goal of the paper is calculation and experimental determination of the average geometrical features of the distribution of macroscopic EWP in circular cylindrical metallic conductors with pulsed axial current of high density.

1. Definition of the problem of the EWP distribution in the metal conductor with pulsed current. We consider a cylindrical coordinate system fixedly positioned in the air straight solid non-magnetic metallic conductor of radius $r_{0}$ and length $l_{0}>>r_{0}$ (Fig. 1). Let air environment parameters correspond to normal atmospheric conditions (air pressure is $1.013 \cdot 10^{5}$ and the temperature $T_{0}$ equals to $\left.0{ }^{\circ} \mathrm{C}[3]\right)$. We consider that the opposite ends of the considered conductor with the conductivity $\gamma_{0}$ of its material applied electrical potential difference, unipolar varying in time $t$ by an arbitrary law. Let us assume that the investigated conductor of crosssection $S_{0}=\pi r_{0}{ }^{2}$ along its longitudinal axis $O Z$ takes pulsed unipolar conduction current $i_{0}(t)$ characterized by the average density $\delta_{0}(t)$. We restrict ourselves to the case when the thickness of the skin layer of the current $\Delta_{i}$ in the conductor material substantially greater than its radius $r_{0}$. For an approximate description of the behavior of electrons drifting free guide use the known single-electron Hartree-Fock approximation which despises their mutual influence on each other, as well as the influence of ions (nodes) of the conductor material of the crystal lattice in his itinerant electrons $[2,3]$. Let the longitudinal and radial distribution of the free electrons of the conductor will be determined on the assumption that their spatial distribution movement and accordingly to the coordinates $\mathrm{r}$ and $\mathrm{z}$ in the first approximation subject to a onedimensional wave temporal Schrödinger equation [3]. Required quantum mechanical based approach in view of the approximate form obtained in $[1,2,5]$ information about the longitudinal and radial distributions of wave drift of free electrons in a conductor under consideration to determine the spatial distribution of average characteristics in its electrically conductive material of uniform periodic quantized longitudinal and radial EWP.

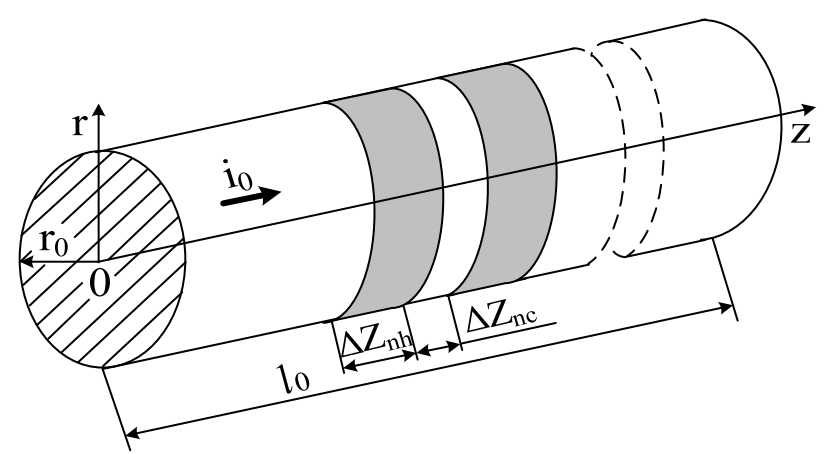

Fig. 1. A solid metal conductor of radius $r_{0}$ and length $l_{0}$ with pulsed axial current $i_{0}(t)$ of high density containing quantized «hot» of width $\Delta z_{n h}$ and «cold» of width $\Delta z_{n c}$ longitudinal sections [2]

2. Selection of the average values of the quantum number for the wave psi-functions of drifting electrons of the metal conductor with pulsed current. For the quantized electron de Broglie half waves propagating along the length $l_{0}$ and the radius $r_{0}$ of the test conductor with a pulsed axial current $i_{0}(t)$ are carried out following known analytical expressions [2]:

$$
\begin{aligned}
& \lambda_{\text {enz }} / 2=l_{0} / n ; \\
& \lambda_{\text {enr }} / 2=r_{0} / n,
\end{aligned}
$$

where $\lambda_{e n z}=h /\left(m_{e} v_{e n z}\right)$ is quantized wave length of the longitudinal drift of a free electron with the rest mass $m_{e}=9.109 \cdot 10^{-31} \mathrm{~kg}[3] ; v_{e n z}=n h /\left(2 m_{e} l_{0}\right)$ is the quantized longitudinal velocity of a free electron drift; $\lambda_{\text {enr }}=h /\left(m_{e} v_{\text {enr }}\right)$ is the quantized wave length of the longitudinal drift of free electron; $v_{e n r}=n h /\left(2 m_{e} r_{0}\right)$ is the quantized radial velocity drift of free electron in the conductor material; $h=6.626 \cdot 10^{-34} \mathrm{~J} \cdot \mathrm{s}$ is the Planck constant [3].

Using (1) and knowing the limits of variation of the quantum number $n=1,2,3, \ldots, n_{m}$, we determine the beginning of the test the average length of the longitudinal conductor electron half-wave $\lambda_{\text {enz }}^{*} / 2$ as follows [9]:

$$
\lambda_{\text {enz }}^{*} / 2=l_{0}\left(n_{m}-1\right)^{-1} \int_{1}^{n_{m}} n^{-1} d n=l_{0}\left(n_{m}-1\right)^{-1} \ln n_{m}
$$

From (2), similar to (3) for the average length of the radial half-wave electronic de Broglie $\lambda^{*}{ }_{\text {enr }} / 2$ or step EWP 
macroscopic radial structure in the electrically conductive material of the considered round conductor we find:

$$
\lambda_{\text {enr }}^{*} / 2=r_{0}\left(n_{m}-1\right)^{-1} \ln n_{m} \text {. }
$$

From (1)-(4) we see that for a quantum number $n$ its average value $\bar{n}$ satisfying the relations $\lambda_{\text {enz }}^{*} / 2=l_{0} / \bar{n}$ and $\lambda^{*}{ }_{e n r} / 2=r_{0} / \bar{n}$ can be written in the following analytical form [10]:

$$
\bar{n}=\left(n_{m}-1\right) /\left(\ln n_{m}\right) .
$$

From (5) for copper, zinc and steel wire $\left(n_{k}=4\right.$ [3]; $n_{m}=2 n_{k}^{2}=32$ [2]) with pulsed current $i_{0}(t)$ it follows that the average value for the quantum numbers $n$ is determined by averaging the fashion wave psi-function in each periodic quantized longitudinal and radial EWP for such conductive materials, will be approximately equal $\bar{n}=9$. This number $\bar{n}$ will correspond to the same average number of «hot» and «cold» longitudinal and radial portions, the amount of average widths which $\left(\Delta z^{*}{ }_{n h}+\Delta z^{*}{ }_{n c}\right)$ and $\left(\Delta r^{*}{ }_{n h}+\Delta r_{n c}^{*}\right)$ formed in the material of the conductor averaged steps periodic structures longitudinal and radial EWP. Below, in section 5 will be given an experimental study on the proposed settlement choice (5) of the numerical value for the number in relation to the above conductive materials investigated current-carrying conductors.

3. Average geometric features for periodic quantized longitudinal EWP in the metal conductor with pulsed current. For the marginal zones of the test metal conductor adjacent to the places of its fastening in high-current circuit with a pulsed current $i_{0}(t)$ and containing longitudinal EWP, taking into account (1) is satisfied the following relation [5]:

$$
\lambda_{\text {enz }}^{*} / 2=\Delta z_{n h}^{*}+2 \Delta z_{n c e}^{*}=l_{0} / \bar{n},
$$

where $\Delta z^{*}{ }_{n h}, \Delta z^{*}{ }_{n c e}$ are the respectively the average values of the quantized width «hot» and extreme «cold» longitudinal sections EWP conductor.

For internal conductor zones adjacent to the above its marginal zones or distant from their averaged geometric characteristics of the quantized longitudinal EWP will be described by the following analytical expression [5]:

$$
\lambda_{\text {enz }}^{*} / 2=\Delta z_{n h}^{*}+\Delta z_{n c i}^{*}=l_{0} / \bar{n},
$$

where $\Delta z^{*}{ }_{n c i}$ is the average value of the quantized width internal «cold» longitudinal sections EWP conductor.

From (6) and (7) shows that the average quantized widths $\Delta z^{*}{ }_{n c i}=\Delta z_{n c}^{*}$ of internal «cold» longitudinal sections of EWP twice the average quantized width $\Delta z^{*}$ nce of respective extremes of «cold» longitudinal sections conductor. To determine the estimated taking into account (6) and (7) of the averaged quantized values $\Delta z^{*}{ }_{n c e}$ and $\Delta z^{*}{ }_{n c i}$ it is necessary to find the average value of the quantized value of $\Delta z^{*}{ }_{n h}$. To this end, in relation to the «hot» longitudinal section of EWP we use the fundamental principle of quantum electrodynamics (mechanics) - Heisenberg uncertainty as follows [3]:

$$
\Delta z_{n h}^{*} \cdot \Delta p_{n z}^{*} \geq h /(4 \pi)
$$

where $\Delta p_{n z}^{*}=m_{e} v_{e n z}^{*}=\bar{n} h /\left(2 l_{0}\right)$ is the averaged quantized longitudinal projection of the momentum of drifting in the microstructure of the conductor of free electrons; $v^{*}{ }_{\text {enz }}=\bar{n} h /\left(2 m_{e} l_{0}\right)$ is the quantized average longitudinal velocity of electrons drifting free conductor.

Then from (8) for the smallest quantized value of the average width $\Delta z^{*}{ }_{n h}$ of «hot» EWP longitudinal portion of the conductor with pulsed axial current $i_{0}(t)$ of high density we have:

$$
\Delta z_{n h}^{*}=l_{0} /(2 \pi \bar{n})=l_{0} \ln n_{m} /\left[2 \pi\left(n_{m}-1\right)\right] .
$$

From (6), taking into account (9) for the averaged quantized widths $\Delta z^{*}{ }_{n c e}$ of extreme «cold» longitudinal sections EWP of the test conductor in the approximate form we get the estimated relation of the form:

$$
\Delta z_{n c e}^{*}=l_{0} \ln n_{m}(2 \pi-1) /\left[4 \pi\left(n_{m}-1\right)\right] .
$$

From (7) and (9) for the average quantized widths $\Delta z^{*}{ }_{n c i}$ of internal «cold» longitudinal sections EWP of the considered conductor we obtain the following approximate ratio for calculations:

$$
\Delta z_{n c i}^{*}=l_{0} \ln n_{m}(2 \pi-1) /\left[2 \pi\left(n_{m}-1\right)\right] .
$$

As a result, the average step for the longitudinal EWP of the test conductor of the periodic structure taking into account (9)-(11) can be determined from the following calculation expression:

$$
\Delta z_{n h}^{*}+2 \Delta z_{n c e}^{*}=\Delta z_{n h}^{*}+\Delta z_{n c i}^{*}=l_{0} \ln n_{m} /\left[\left(n_{m}-1\right)\right] \text {. }
$$

4. Average geometric characteristics for periodic quantized radial EWP in the metal conductor with pulsed current. For the central and outer zones of the cylindrical conductor of radius $r_{0}$ with current $i_{0}(t)$ containing radial EWP using (2) we have:

$$
\lambda_{e n r}^{*} / 2=\Delta r_{n h}^{*}+2 \Delta r_{n c e}^{*}=r_{0} / \bar{n},
$$

where $\Delta r_{n h}^{*}, \Delta r^{*}{ }_{n c e}$ are the respectively the average values of the quantized width «hot» and extreme «cold» areas of radial conductor EWP.

For internal circular zones of the considered conductor radial averaged geometrical characteristics of EWP satisfy the following approximate calculated ratio $[5]$ :

$$
\lambda_{\text {enr }}^{*} / 2=\Delta r_{n h}^{*}+\Delta r_{n c i}^{*}=r_{0} / \bar{n},
$$

where $\Delta r_{n c i}^{*}$ is the the average value of the quantized width internal «cold» curved sections EWP of the conductor.

We write further fundamental Heisenberg uncertainty for localized to the «hot» areas of EWP radial drift of free electrons in the form [3]:

$$
\Delta r_{n h}^{*} \cdot \Delta p_{n r}^{*} \geq h /(4 \pi)
$$

where $\Delta p_{n r}^{*}=m_{e} v^{*}{ }_{e n r}=\bar{n} h /\left(2 r_{0}\right)$ is the quantized average radial projection pulse drifting in the microstructure of the test conductor free electrons; $v_{e n r}^{*}=\bar{n} h /\left(2 m_{e} r_{0}\right)$ is the quantized average radial velocity drift of free electrons in a circular cylindrical conductor. 
From (15) and (5) for the smallest quantized value of the average width $\Delta r_{n h}^{*}$ of «hot» radial section of EWP test conductor with a pulsed current $i_{0}(t)$ of high density we obtain:

$$
\Delta r_{n h}^{*}=r_{0} /(2 \pi \bar{n})=r_{0} \ln n_{m} /\left[2 \pi\left(n_{m}-1\right)\right] .
$$

As a result of (13) and (16) for the average quantized widths $\Delta r^{*}{ }_{n c e}$ of extreme «cold» areas of the considered radial conductor EWP in the adopted approximation we find:

$$
\Delta r_{n c e}^{*}=r_{0} \ln n_{m}(2 \pi-1) /\left[4 \pi\left(n_{m}-1\right)\right] .
$$

From (14) with (16) to the averaged quantized widths $\Delta r^{*}{ }_{n c i}$ of internal «cold» radial sections EWP of the test conductor write the following calculation ratio:

$$
\Delta r_{n c i}^{*}=r_{0} \ln n_{m}(2 \pi-1) /\left[2 \pi\left(n_{m}-1\right)\right] .
$$

Then, for the average pitch of the periodic localization of free electrons drifting in the «hot» areas of EWP along the outer radius $r_{0}$ of the considered conductor (16)-(18) we have:

$$
\Delta r_{n h}^{*}+2 \Delta r_{n c e}^{*}=\Delta r_{n h}^{*}+\Delta r_{n c i}^{*}=r_{0} \ln n_{m} /\left[\left(n_{m}-1\right)\right] \text {. }
$$

From (9)-(12) and (16)-(19) that have been found in this approximation averaged geometrical dimensions of the «hot» and «cold» areas of radial EWP in $l_{0} / r_{0}$ times smaller than the corresponding average sizes for longitudinal EWP conductor pulse current $i_{0}(t)$ of high density. This feature in the spatial distribution of longitudinal and radial EWP solid round conductor is important for a number of advanced electric technologies [11] using the flow of metal conductors for large pulsed currents with a view to their electrical explosion (EE). The most fully, it will be shown on receipt by EE gases in the thin metal wires of small «metal» of the plasma, and then, after separation of plasma nanoproducts and microdispersed conductive materials [12].

5. The results of experimental verification of choice for the average features of quantized longitudinal EWP in the metal conductor with pulsed current. To verify (the truth test) selection by (5) the average value of the quantum number $n$ and the proposed settlement of the relations (9)-(12) with respect to the averaged quantized characteristics of longitudinal EWP in the test conductor use presented in $[1,2,5]$, the results of experimental studies wave longitudinal distribution of free electrons and drifting respectively, «hot» and «cold» longitudinal sections in galvanized steel wire $\left(r_{0}=0.8 \mathrm{~mm}\right.$; $\left.l_{0}=320 \mathrm{~mm} ; S_{0}=2.01 \mathrm{~mm}^{2} ; n_{k}=4\right)$ with the thickness of the outer protective coating $\Delta_{0}=5 \mu \mathrm{m}$ was tested in the discharge circuit of a powerful high voltage generator prolonged $C$ - components of artificial lightning current [13] a direct impact aperiodic current pulse $i_{0}(t)$ of temporary shape $t_{m} / \tau_{p}=9 \mathrm{~ms} / 160 \mathrm{~ms}$ of large average density $\delta_{0 m} \approx I_{\mathrm{o} m} / S_{0} \approx 0.37 \mathrm{kA} / \mathrm{mm}^{2}$. This corresponds to the maximum value of the amplitude $\delta_{0 m}$ unipolar current pulse $I_{\mathrm{o} m}=745 \mathrm{~A}$, the onset of which is equal to $t_{m}=9 \mathrm{~ms}$. On $0.5 I_{\mathrm{o} m}$ level measurements made in accordance with the help of an attorney in the metrology service coaxial measuring shunt type ШК-300, which has for the mode conversion factor of $56.42 \cdot 10^{2} \mathrm{~A} / \mathrm{V}[2,13]$, the duration used in the experiments conducted by the pulse current was $\tau_{p}=160 \mathrm{~ms}$, and the total duration of the flow through the wire of said current pulse in the partial destruction of its metal structure of intense Joule heating of nonmagnetic material wire reached about $576 \mathrm{~ms}[1,2]$. Note that in the present case for the current round of the skin layer of the steel wire in a stationary mode, you kind of relation $[2,6]: \quad \Delta_{i} / r_{0} \approx 2 r_{0}{ }^{-1}\left[t_{m} /\left(\pi \mu_{0} \gamma_{0}\right)\right]^{1 / 2} \approx 42$, where $\mu_{0}=4 \pi \cdot 10^{-7} \mathrm{H} / \mathrm{m}$ is the magnetic constant [3] and $\gamma 0 \approx$ $\gamma_{0} \approx 8 \cdot 10^{6} \mathrm{~S} / \mathrm{m}$ is the conductivity base wire used in experiments [3]). Running for $\Delta_{i} / r_{0}$ of the specified quantitative value may be indicative of the appropriateness of the estimates for the amplitude of the average current density $\delta 0 \mathrm{~m}$, for which the amplitude and temporal parameters (ATP) test aperiodic pulse type 9 $\mathrm{ms} / 160 \mathrm{~ms}$ current steel wire taken from the corresponding experimental waveforms cited in [2].

Fig. 2 shows the results of electro-thermal effect to the selected contact steel wire aperiodic current pulse 9 $\mathrm{ms} / 160 \mathrm{~ms}$ with indicated ATP $\left(I_{\mathrm{o} m}=745 \mathrm{~A} ; t_{m}=9 \mathrm{~ms} ; \tau_{p}\right.$ $=160 \mathrm{~ms})$. It can be seen that in this case $\left(\delta_{0 m} \approx 0.37\right.$ $\mathrm{kA} / \mathrm{mm}^{2}$ ) along the longitudinal axis of galvanized steel wire are four sphere-like «hot» (quantized $\Delta z_{n h} \approx 7 \mathrm{~mm}$ width) and two cylindrical internal «cold» (mm quantized width $\Delta z_{n c i} \approx 27$ ) longitudinal sections of EWP. The other five «hot» and eight «cold» (of which the two extreme of width $\Delta z_{n c e}=\Delta z_{n c i} / 2$ experimentally confirmed in accordance with this ratio in [14]) longitudinal sections of EWP wire undergone a complete sublimation. The specified number of «hot» and «cold» sites of investigated EWP longitudinal wires can say that in this case the experimental stochastic quantum number $\mathrm{n}$, the equality $n=\bar{n}=9$. From the calculated ratio (9) at $n_{m}=2 n_{k}{ }^{2}=32$ and, accordingly, $\bar{n}=9$ that the value of the average width of the «hot» area of the longitudinal steel wire numerically EWP is about $\Delta z^{*}{ }_{n h}=5.7 \mathrm{~mm}$ (with the experimental value of this width $\Delta z_{n h} \approx 7 \mathrm{~mm}$ [1]). It is important to emphasize that found on (9) the average width $\Delta z^{*}{ }_{n h}$ of «hot» EWP longitudinal portion substantially corresponds to the numerical value of the quantized width $\Delta z_{n h}$ of this site of EWP of indicated conductor, as defined in $[1,2,5]$ on the following calculation relationship:

$$
\Delta z_{n h}=e_{0} n_{e 0} h\left(m_{e} \delta_{0 m}\right)^{-1}\left[8+(\pi-2)^{2}\right]^{-1},
$$

where $e_{0}=1.602 \cdot 10^{-19} \mathrm{~K}$ is the module of the electron electric charge; $n_{e 0}$ is the average density of free electrons in the metal conductor considered prior to flowing through it a current pulse $i_{0}(t)$.

From (20) obtained in $[2,5]$ based on the fundamental Heisenberg uncertainty relation [3] we have used for the steel wire at $16.82 \cdot 10^{28} \mathrm{~m}^{-3}[2,3]$ and $\delta_{0 m} \approx 0.37 \cdot 10^{9} \mathrm{~A} / \mathrm{m}^{2}$ it follows that $\Delta z_{n h} \approx \Delta z^{*}{ }_{n h} \approx 5.7 \mathrm{~mm}$. These calculated width «hot» EWP longitudinal portions within $19 \%$ different from the previously experimentally obtained in [1] for the case of electro widths $\left(\Delta z_{n h} \approx 7 \mathrm{~mm}\right)$ 
respective portions of said wire. It can be reasonably concluded that the quantized width $\Delta z_{n h}$ of «hot» longitudinal portion EWP of the metal conductor according to (20) is also an averaged geometrical feature of the longitudinal EWP. Its numerical value at a constant value in the test conductor $\delta_{0 m}$ also remains unchanged, as evidenced by the results carried out at the Scientific-\&Research Planning-\&-Design Institute «Molniya» of the NTU «KhPI» high-temperature experiments using such a steel wire $\left(r_{0}=0.8 \mathrm{~mm} ; l_{0}=320 \mathrm{~mm} ; \Delta_{0}=5 \mu \mathrm{m} ; S_{0}=2.01\right.$ $\left.\mathrm{mm}^{2}\right)$ [1, 2, 5]. The above computational and experimental data for the width of the EWP $\Delta z_{n h}$ indicate the validity of the selection to (5), taking into account the relation $n_{m}=2 n_{k}^{2}$ of the average value $\bar{n}$ for the whole of the integer $n$.

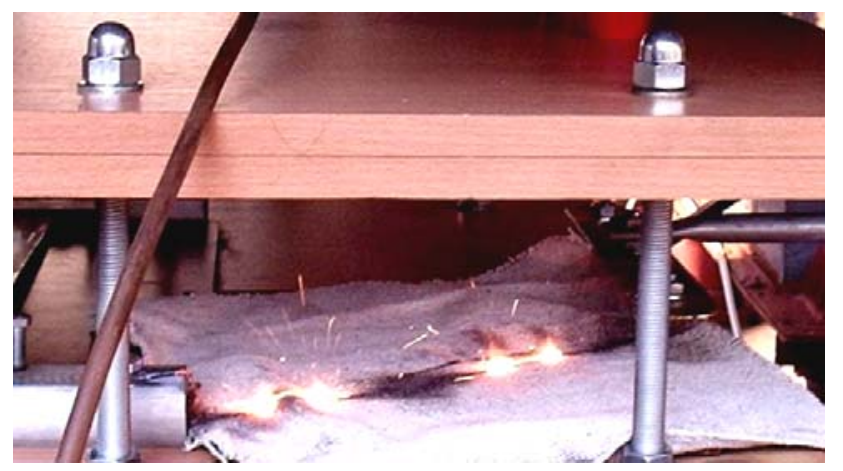

Fig. 2. External view of cooling in the air and asbestos cloth quantized sphere-like «hot» (width $\Delta z_{n h} \approx 7 \mathrm{~mm}$ ) and cylindrical inner «cold» (width $\Delta z_{n c i} \approx 27 \mathrm{~mm}$ ) longitudinal sections (macroscopic areas of quantized longitudinal EWP) galvanized steel wire $\left(r_{0}=0.8 \mathrm{~mm} ; l_{0}=320 \mathrm{~mm} ; \Delta_{0}=5 \mu \mathrm{m} ; S_{0}=2.01 \mathrm{~mm}^{2}\right)$ immediately after exposure to an aperiodic pulsed axial current $i_{0}(t)$ of the temporary shape $t_{m} / \tau_{p}=9 \mathrm{~ms} / 160 \mathrm{~ms}$ of high density

$$
\begin{gathered}
\left(I_{\mathrm{o} m}=745 \mathrm{~A} ; t_{m}=9 \mathrm{~ms} ; \tau_{p}=160 \mathrm{~ms}\right. \\
\left.\delta_{0 m} \approx 0.37 \mathrm{kA} / \mathrm{mm}^{2} ; n=\bar{n}=9\right)[1]
\end{gathered}
$$

With regard to the average quantized width $\Delta z^{*}{ }_{n c i}$ of internal «cold» longitudinal sections EWP of the steel wire, it is, according to (11) at $n_{m}=32$ and $\bar{n}=9$ and taking into account (5) numerically is about $29.9 \mathrm{~mm}$ (experimental value of this width $\Delta z_{n c i} \approx 27 \mathrm{~mm}$ [1]). It is evident that the design for (11) the average value of width $\Delta z_{n c i}$ of internal «cold» EWP longitudinal section for the considered steel wire differs from the experimentally obtained it widths of longitudinal EWP $\Delta z_{n c i}$ corresponding portion within $10 \%$.

To carry out even in rough form on a powerful highvoltage high-current electrical installation verification of the calculated relations (16)-(19) with respect to the averaged quantized geometric characteristics of radial EWP in the study of metallic conductors with an axial current pulse $i_{0}(t)$ of high density now practically it is not possible due to lack of the necessary high-speed photorecording equipment. We hope for their experimental verification in future in the years to its own high current research or prospecting work of other scientists in the area of electrical physics.

\section{Conclusions.}

1. In the framework of quantum-mechanical approach to the electric properties of high density periodic EWP of macroscopic dimensions arising from the pulsed axial current in circular metallic conductors certain scientific generalizations are carried out and calculation relations (3)-(5) are proposed for an approximate determination of the average value $\bar{n}$ of integer quantum number $n$ for propagating their electrically conductive material quantized modes of wave psi-functions of the $n$-th order and the quantized electron de Broglie half-waves and, based on the calculated ratio obtained by (9)-(12) and (16)-(19) for estimating the average geometric features of longitudinal and radial EWP periodically placed along the length $l_{0}$ and radius $r_{0}$ of investigated conductors.

2. Experimental verification on a powerful high-current high-voltage generator long-term aperiodic $C$ - artificial lightning current component of the results of the proposed selection by (5) of the average value $\bar{n}$ of integer quantum number $n$ and approximate calculation according to (9)-(12) of averaged geometrical features of quantized longitudinal macroscopic EWP in continuous galvanized steel wire $\left(r_{0}=0.8 \mathrm{~mm} ; l_{0}=320 \mathrm{~mm} ; \Delta_{0}=5 \mu \mathrm{m} ; S_{0}=2.01\right.$ $\mathrm{mm}^{2}$ ) with aperiodic pulse temporal shape of the current 9 $\mathrm{ms} / 160 \mathrm{~ms}$ of high density $\left(I_{\mathrm{o} m}=745 \mathrm{~A} ; t_{m}=9 \mathrm{~ms} ; \tau_{p}=\right.$ $160 \mathrm{~ms}, \delta_{0 m} \approx 0.37 \mathrm{kA} / \mathrm{mm}^{2}$ ) confirmed their authenticity.

3. Verification of the calculation relations (16)-(19) for the average geometric features of the quantized radial EWP in electrically exploding metal conductors with high pulsed axial current requires to carry out in future complex and expensive experimental electrophysical studies in a high-voltage laboratory.

\section{REFERENCES}

1. Baranov M.I. Features heating thin bimetallic conductor large pulse current. Elektrichestvo, 2014, no.4, pp. 34-42. (Rus).

2. Baranov M.I. The main characteristics of the wave distribution of free electrons in a thin metallic conductor with a pulse current of high density. Elektrichestvo, 2015, no.10, pp. 20-32. (Rus).

3. Kuz'michev V.E. Zakony i formuly fiziki [Laws and formulas of physics]. Kiev, Naukova Dumka Publ., 1989. 864 p. (Rus).

4. Baranov M.I. Local heating of electrical pathways of power electrical equipment under emergency conditions and overcurrents. Russian Electrical Engineering, 2014, vol.85, no.6, pp. 354-357. doi: 10.3103/s1068371214060030.

5. Baranov M.I. Quantum-wave nature of electric current in a metallic conductor and some of its electrophysical macrophenomena. Electrical engineering \& electromechanics, 2014, no.4, pp. 25-33. doi: 10.20998/2074-272X.2014.4.05.

6. Baranov M.I. Izbrannye voprosy elektrofiziki. Tom 2, Kn. 2: Teoriia elektrofizicheskikh effektov $i$ zadach [Selected topics of Electrophysics. Vol.2, Book 2. A theory of electrophysical effects and tasks]. Kharkiv, NTU «KhPI» Publ., 2010. 407 p. (Rus).

7. Orlov I.N. Elektrotehnicheskij spravochnik. Proizvodstvo $i$ raspredelenie elektricheskoj energii. Tom 3, Kn. 1 [Electrical engineering handbook. Production and distribution of electric energy. Vol. 3, Book 1. Ed. I.N. Orlov]. Moscow, Energoatomizdat Publ., 1988. 880 p. (Rus). 
8. Baranov M.I., Kravchenko V.I. Electrothermal resistance wire and cable to the aircraft to the striking action pulsed current lightning. Elektrichestv, 2013, no.10, pp. 7-15. (Rus).

9. Dwight H.B. Tablicy integralov $i$ drugie matematicheskie formuly [Tables of integrals and other mathematical data]. Moscow, Nauka Publ., 1973. 228 p. (Rus).

10. Baranov M.I., Rudakov S.V. Averaged characteristics of the wave drifting distribution of electrons in a metal conductor with a pulse current conduction of high density. Bulletin of NTU «KhPI», 2013, no.60(1033), pp. 12-20. (Rus).

11. Guly G.A. Nauchnye osnovy razrjadno-impul'snyh tehnologij [Scientific basis of the discharge-pulse technology]. Kiev, Naukova dumka Publ., 1990. 208 p. (Rus).

12. Baranov M.I. Preparation of disperse materials with micron, submicron and nanostructured matter particles under electric explosion of thin metallic conductors. Electrical engineering \& electromechanics, 2012, no.4, pp. 45-49. (Rus). doi: 10.20998/2074-272X.2012.4.09.

13. Baranov M.I., Koliushko G.M., Kravchenko V.I., Nedzel'skii O.S., Dnyshchenko V.N. A Current Generator of the Artificial Lightning for Full-Scale Tests of Engineering Objects. Instruments and Experimental Technique, 2008, no.3, pp. 401405. doi: 10.1134/s0020441208030123.
14. Baranov M.I. Theoretical and experimental results of research into explanation of de Broglie half-wave existence in the microstructure of an active metallic conductor. Electrical engineering \& electromechanics, 2014, no.3, pp. 45-49. (Rus). doi: 10.20998/2074-272X.2014.3.09.

Received 25.05.2016

M.I. Baranov ${ }^{1}$, Doctor of Technical Science, Chief Researcher, S.V. Rudakov ${ }^{2}$, Candidate of Technical Science, Associate Professor,

${ }^{1}$ Scientific-\&-Research Planning-\&-Design Institute «Molniya», National Technical University «Kharkiv Polytechnic Institute», 47, Shevchenko Str., Kharkiv, 61013, Ukraine.

${ }^{2}$ National University of Civil Protection of Ukraine, 94, Chernyshevska Str., Kharkiv, 61023, Ukraine. phone +38057 7076841, e-mail: eft@kpi.kharkov.ua, serg 73@i.ua

How to cite this article:

Baranov M.I., Rudakov S.V. Average geometrical features of the electron wave packages distribution in metallic conductors with pulsed axial current of high density. Electrical engineering \& electromechanics, 2016, no.5, pp. 29-34. doi: 10.20998/2074-272X.2016.5.04. 